\title{
Membrane fouling control and cleaning technology of ceramic membrane treating wastewater
}

\author{
Guoqiang $\mathrm{Ma}^{1}$, Shoubin Zhang ${ }^{1, *}$, Yanming Yang ${ }^{2}$, Liping Qiu ${ }^{1}$, Guicai Liu ${ }^{1}$, Jingxiu Zhong ${ }^{1}$ \\ ${ }^{1}$ School of Civil Engineering \& Architecture, University of Jinan, NO.336, Nanxinzhuang West Road., Jinan 250022, P.R.China \\ ${ }^{2}$ Shandong Science and Technology Statistical Analysis Research Center. NO.607, Shunhua Road., Jinan 250101, P.R.China
}

\begin{abstract}
Ceramic membrane technique was a new and efficient technology for wastewater treatment and used more and more widely in recent years. Controlling membrane fouling was the key method to ensure the efficient and stable operation of ceramic membrane. In this paper, the causes, influencing factors and control methods of ceramic membrane fouling were summarized. As one of the most effective means to control membrane fouling, several common membrane cleaning methods, such as physical cleaning, chemical cleaning, ultrasonic cleaning and combined cleaning, were introduced. And the application of ceramic membrane cleaning was presented too. Then the future directions for ceramic membrane techniquresearching was prospected.
\end{abstract}

\section{Introduction}

The ceramic membrane is an inorganic porous membrane formed by a special process of materials such as $\mathrm{SiO}_{2}$, $\mathrm{Al}_{2} \mathrm{O}_{3}$, and $\mathrm{TiO}_{2}$. Compared with organic film, ceramic film has the characteristics of high temperature resistance, corrosion resistance, strong chemical stability and high mechanical strength ${ }^{[1]}$. As the running time goes on, membrane fouling will inevitably occur, and the membrane flux of the ceramic membrane will decrease significantly, which will affect the water treatment efficiency and shorten the life expectancy, thus affecting the use. Therefore, how to control membrane fouling and find the optimal cleaning method to improve the operating efficiency of the membrane has become an important research direction in recent years.

\section{Ceramic membrane contamination}

Membrane fouling is mainly caused by solute macromolecules, particles and colloids deposited in the raw water on the surface of the membrane or adsorbed inside the membrane pores, which will partially or even completely block the membrane pores, resulting in a decrease in membrane flux, a decrease in separation performance, and a deterioration in effluent quality. The pollution of ceramic membrane is divided into physical pollution and chemical pollution ${ }^{[2]}$. The membrane fouling at the initial stage of filtration is mainly caused by concentration polarization ${ }^{[3]}$. Since the pores of the membrane are selective, molecules or particles larger than the pores of the membrane cannot pass through themembrane, and are trapped on the surface of the membrane, and deposit on the surface of the membrane over time. The aggregates eventually form a gel layer, which deteriorates the separation characteristics of the membrane and the quality of the effluent water, resulting in flaws in the membrane system.

\subsection{Factors affecting ceramic membrane fouling}

(1) The film's own resistance. This part of the resistance comes from the properties of the ceramic membrane itself, including its membrane material, pore size, hydrophilicity of the membrane surface, and the like.

(2) Concentration polarization layer resistance. Due to the selective permeability of the membrane, the trapped component accumulates on the liquid side surface of the membrane, and the osmotic pressure of the boundary layer increases, so that the permeation flux of the membrane decreases. This part of the concentration polarization resistance is reversible and can usually be overcome by optimizing the control parameters ${ }^{[4]}$.

(3) Gel layer resistance. This part of the resistance is caused by the continuous accumulation of contaminant particles on the membrane surface to achieve precipitation and deposition, which needs to be removed by backwashing or chemical cleaning. In activated sludge [5], the membrane fouling resistance is mainly composed of gel layer resistance, accounting for $50 \%-90 \%$ of the total resistance. The increase of resistance is mainly due to the blockage resistance of the membrane pores.

\section{2 control method of ceramic membrane pollution}

The control methods of ceramic membrane pollution can be divided into three categories:

\footnotetext{
* Corresponding author: cea_zhangsb@ujn.edu.cn
} 
Hydrophilic modification of the ceramic membrane, such as coating a hydrophilic material on the surface of the ceramic membrane to obtain a ceramic membrane material having higher hydrophilicity, so that pollutants are not easily deposited on the surface of the membrane [6].

(2) Before the ceramic membrane treatment, the corresponding pretreatment equipment can be added to reduce the type and quantity of pollution, so as to maintain the relative stability of the influent water quality. Or change the characteristics of pollutants in the influent, and thus effectively control the contamination of the ceramic membrane.

(3) Change the operation control parameters of the ceramic membrane. The operating control parameters can have a direct impact on the operating flux of the ceramic membrane, where filtration events, batch times, backwashing times, etc. have a significant effect on the change in membrane flux during operation of the ceramic membrane. In practical applications, optimizing operating parameters is the most effective way to slow down membrane fouling.

\section{Ceramic membrane fouling cleaning technology}

The contamination of the ceramic membrane is unavoidable during operation. In order to restore the performance parameters of the film as much as possible, prolong the service life of the film, and reduce the cost of replacing the film, We must clean the ceramic membrane regularly.

\subsection{Physical cleaning}

The physical cleaning method has the characteristics of not introducing new pollutants, simple cleaning steps, etc., and can remove a variety of pollutants, but the cleaning is not thorough enough ${ }^{[7]}$. However, this method is effective only in the initial stage of contamination of the ceramic membrane, and the cleaning effect rapidly decreases with time.

Backwashing: This is one of the most common cleaning methods. Backwashing uses a liquid or gas as a back-up carrier, applies pressure to the ceramic membrane in the direction of the permeate, and cleans out the contaminants present in the membrane and in the pores, thereby achieving the effect of restoring the membrane flux ${ }^{[8]}$.

Mechanical scraping: This method requires the use of a medium such as a soft foam ball and a sponge ball to wash the film multiple times by water pressure, and then achieve the goal of removing impurities ${ }^{[9]}$. This method has a good cleaning effect on organic rubber impurities.

Negative pressure cleaning: By vacuum suction, a negative pressure is formed on the functional side of the membrane to remove surface contaminants and contaminants in the pores of the membrane.
When the pollution is serious, the physical cleaning is almost ineffective, and chemical cleaning is required. Chemical cleaning involves placing a contaminated ceramic membrane into a specific chemical cleaning agent that chemically reacts with contaminants to remove contaminants from the surface of the ceramic membrane or pores of the membrane to restore membrane flux. Common chemical cleaning agents are built in Table 1.

Table 1. Types of cleaning agents and their functions

\begin{tabular}{|c|c|c|}
\hline $\begin{array}{c}\text { Type of } \\
\text { cleanin } \\
\text { g agent }\end{array}$ & $\begin{array}{c}\text { Main } \\
\text { case }\end{array}$ & Features \\
\hline Acid & $\begin{array}{c}\mathrm{HCl} \\
\mathrm{H}_{2} \mathrm{C}_{2} \mathrm{O}_{4}\end{array}$ & $\begin{array}{c}\text { Removal of inorganic salt } \\
\text { precipitates from the membrane } \\
\text { surface mainly by } \\
\text { neutralization reaction and } \\
\text { metathesis reaction }\end{array}$ \\
\hline Alkali & $\begin{array}{c}\mathrm{NaOH} \\
\mathrm{KOH}\end{array}$ & $\begin{array}{c}\text { Removal of organic pollutants } \\
\text { from the membrane surface by } \\
\text { hydrolysis and dissolution }\end{array}$ \\
\hline Oxid]
\end{tabular}

\subsection{Other cleaning}

Ultrasonic cleaning: Ultrasonic cleaning is the direct and indirect effect of the cavitation, acceleration and direct flow of the ultrasonic wave on the liquid, so that the dirt layer is dispersed, emulsified and stripped to achieve the cleaning purpose.Ultrasonic cleaning has the advantages of good cleaning effect and fast cleaning speed.

Combination cleaning: When the single agent cleaning effect is weak, it can be considered to use different drugs in combination. Different cleaning agents, combined with compounding, can simplify the cleaning step and enhance the cleaning effect. Liu Zhenyang et al [3] found that in OP-10, $\mathrm{NaOH}, \mathrm{EDTA}-\mathrm{Na}_{2}, \mathrm{NaClO}$, SDBS, the combined cleaning effect of different agents was higher than the single agent cleaning effect, and the recovery rate of membrane clear water flux was above $90 \%$. It is indicated that there is a clear synergy between the two agents used in the five agent combination cleaning methods.

\subsection{Chemical cleaning}




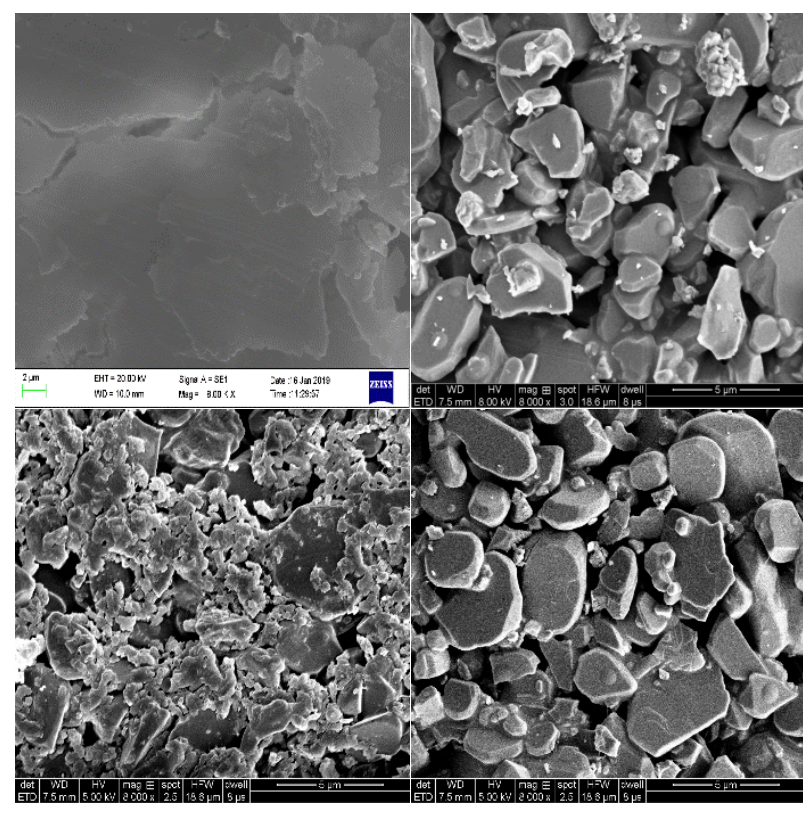

Fig. 1. SEM topography of the film surface

Note: The upper left is the SEM topography of the new film surface; the upper right is the SEM topography of the contaminated film surface without any cleaning; the lower left is the SEM topography of the contaminated film surface after physical cleaning; the lower right is the pollution after chemical cleaning. Membrane surface SEM morphology

\section{Ceramic membrane technology application}

\subsection{Oil production wastewater}

The oil content and solid suspended solids concentration (SS) of the oil production wastewater are high, and direct discharge will cause serious water pollution. The water quality after the conventional treatment method cannot meet the needs of low permeability formation re-injection. Ding Hui et al ${ }^{[11]}$ found that after the membrane was immersed in $0.05 \mathrm{~mol} / \mathrm{L} \mathrm{HCl}$ or $\mathrm{HNO}_{3}$ solution for $12 \mathrm{~h}$, the filtration performance recovered well, and the recovery rate of membrane flux was above $96 \%$, of which $0.05 \mathrm{~mol} / \mathrm{L}$. After the membrane was immersed in $\mathrm{HNO}_{3}$ solution for 12 hours, the membrane flux recovery rate reached $97.2 \%$. It has also been found that a combined cleaning of $\mathrm{NaOH}$ and $\mathrm{HNO}_{3}$ helps to restore membrane flux.

\subsection{Coking wastewater}

The coking wastewater has high oil content and high organic matter concentration. It can be determined that the main pollution sources are oil pollution, organic matter adsorption layer and metal deposition (iron). It is precisely because these substances are adsorbed on the membrane surface, which causes the membrane flux to decay rapidly. Ai Yulian et al ${ }^{[7]}$ found that in the two-step cleaning, $0.6 \% \mathrm{NaClO}$ (the first step) $+1.5 \% \mathrm{NaOH}$ (the second step) had a relatively good cleaning effect, and the membrane flux recovery rate reached $88.3 \%$, which was better than the single-step cleaning effect when $0.6 \%$ $\mathrm{NaClO}$ and $1.5 \% \mathrm{NaOH}$ were used as cleaning agents alone.

\section{Conclusion}

As a new type of water treatment technology, ceramic membrane separation technology has great potential for development. However, due to the inevitable pollution of ceramic membrane and low cleaning efficiency, it has greatly restricted the popularization and application of ceramic membrane water treatment technology, which has greatly restricted The healthy development of the ceramic membrane industry. Therefore, the future research direction is to improve the development of ceramic membranes with higher anti-pollution ability, and to find more efficient and cheap chemical cleaning combination, improve cleaning ability and obtain higher economic benefits.

\section{Acknowledgements}

This work was financially supported by the Shandong Provincial Key Research and Development Program(2017GSF17105).

\section{References}

1. Li Bowen, Li Xiang, Zhou Li and so on. Ozone ceramic membrane process for micro-polluted raw water effect and membrane pollution research $[\mathrm{J}]$. Water treatment technology, 44, 1(2018).

2. Tang Shuwen, Ji Yun, Wang Mengyu, etc. Rural domestic sewage ceramic membrane-bioreactor treatment process to strengthen nitrogen and phosphorus removal research [J]. Journal of AgroEnvironment Science, 38, 5 (2019).

3. Liu Zhenyang. Research on process control and membrane fouling cleaning technology of flat ceramic membrane in Wanggao Wastewater Treatment Plant [D]. Tsinghua University, (2017).

4. Wang Wei, Zhu Xinjian, Hu Yuan et al. Experimental study on reverse osmosis membrane fouling and membrane cleaning of high-salt wastewater $[\mathrm{J}]$. Applied Chemicals, 48, 6 (2019).

5. Li Yansong. Membrane pollution factor identification and gel layer structure analysis [D]. Dalian University of Technology, (2006).

6. Pilot study on decontamination efficiency and membrane fouling control of a new ultrafiltration system [J]. Water supply and drainage, 6, 9 ( 2019).

7. Ai Yulian. Ceramic membrane cleaning process research [J]. Ceramics, 12 (2016).

8. Gao W, Liang H, Ma J, et al. Membrane fouling control in ultrafiltration technology for drinking 
water production: areview[J]. Desalination, 272 (2011).

9. Wang Wei, Zhao Dezhi, Wang Dehui, etc. Ceramic membrane pollution and cleaning technology progress [J]. Applied Chemicals, 47, 6 (2018).

10. Li Qian, Wan Wen. Chemical cleaning of ceramic membrane in cold rolling oily wastewater treatment [J]. Cleaning the world, 34, 2 (2018).

11. Ding Hui, Peng Zhaoyang, Li Yi, etc. Inorganic ceramic membrane treatment of oilfield produced water [J]. Journal of Environmental Engineering, 7,4 (2013). 